\title{
E-Cadherin Is Important for Meibomian Gland Function as Revealed by a New Human ex Vivo Slice Culture Model
}

\author{
Vera Rötzer, ${ }^{*}$ Francesca Melega, ${ }^{*}$ Fabian Garreis, ${ }^{\dagger}$ Friedrich Paulsen, ${ }^{\dagger}$ and Jens Waschke*
}

From the Department of Vegetative Anatomy, * Institute of Anatomy, Ludwig-Maximilians-Universität München, Munich; and the Department of Functional and Clinical Anatomy, ${ }^{\dagger}$ Friedrich-Alexander-University Erlangen-Nürnberg, Erlangen, Germany

\author{
Accepted for publication \\ April 10, 2019. \\ Address correspondence to \\ Jens Waschke, M.D., Institute \\ of Anatomy, Medical Faculty, \\ Department I, LMU Munich, \\ Pettenkoferstraße 11, 80336 \\ Munich, Germany. E-mail: \\ jens.waschke@med.uni- \\ muenchen.de.
}

\begin{abstract}
Meibomian glands within the eyelid are important for the maintenance of the integrity and health of the ocular surface. Patients with the blistering skin disease pemphigus vulgaris (PV), which is caused by autoantibodies against desmosomal cadherins, often have dry eye disease. Therefore, we studied the regulation of cell cohesion in human meibomian gland epithelial cells (HMGECs). During serum-induced differentiation for 1 to 6 days, HMGECs drastically enhanced intercellular cohesion, whereas lipid production did not change. The expression profiles of the desmosomal PV antigens desmoglein (Dsg) 3 and 1 but not of the adherens junction component E-cadherin (Ecad) was dependent on the presence of serum. Surprisingly, after 1 day but not after 6 days of serum-induced differentiation, an inhibitory antibody against Ecad drastically reduced intercellular cohesion and blocked lipid production of HMGECs. In contrast, antibodies against desmosomal cadherins, including human and mouse pemphigus autoantibodies, had no effect on monolayer integrity and lipid production. Because lipid production was unaltered in meibomian glands from Dsg3-deficient mice, we established an ex vivo slice culture model of human eyelids to allow studies in a more physiologic environment. Here, the inhibitory antibody against Ecad but not a Dsg3-specific PV antibody interfered with stimulated lipid production. Together, these data demonstrate that cell cohesion is maintained differently in meibomian gland cells and indicate that Ecad is important for meibomian gland function. (Am J Pathol 2019, 189: 1559-1568; https://doi.org/10.1016/j.ajpath.2019.04.015)
\end{abstract}

Because the meibomian gland dysfunction (MGD) is identified as a main cause of the dry eye disease (DED), ${ }^{1,2}$ molecular mechanisms underlying (patho-)physiologic processes of meibomian glands raised increasing interest in eye research. This holocrine, modified type of sebaceous glands within the upper and lower eyelid contributes substantially to the integrity and health of the ocular surface by producing an oily secretion important to stabilize the tear film and prevent its evaporation. Therefore, meibocytes accumulate lipid vesicles on their centripetal passage through the meibomian gland acini before they decompose and get released into the ductal system. ${ }^{3}$ With the use of human meibomian gland epithelial cells (HMGECs), it was shown that the serum-induced differentiation promoted the formation of keratin filaments and desmosomes. ${ }^{4}$ In an in situ study on human eyelids, the differentiation-dependent distribution pattern of adhesion molecules within meibomian glands was corroborated. Here, meibocytes displayed most desmosomal cadherins, including desmoglein (Dsg), desmocollins, and E-cadherin (Ecad), as the main adhesion molecule of the adherens junction (AJ) but no tight junction components. The clinical relevance of intact intercellular contacts is underscored by diseases such as pemphigus vulgaris (PV) in which autoantibodies are directed against Dsg1 and Dsg3 and cause intraepithelial blistering within the skin and the mucosa as well as DED. ${ }^{6-9}$ However, up to now the regulation of intercellular cohesion among meibocytes and

Supported by Friedrich-Baur-Stiftung grant 01/17 (V.R.), a Deutsche Opthalmologische Gesellschaft stipend (F.M.), and DFG grant DFG FOR2497; PEGASUS (J.W.).

Disclosures: None declared. 
the role for (patho-)physiologic processes, such as cell differentiation and maturation or MGD, have not been investigated.

Because MGD received increased interest with regard to DED, an increasing number of models for studying MGD are available, and the number of publications on meibomian glands has increased over the years. However, at the moment, many in vitro studies are confronted with limitations in reflecting the in vivo situation. ${ }^{4,10,11}$ Moreover, up to now no satisfactory animal model exists that resembles the pathophysiologic aspects of human MGD. ${ }^{11,12}$ Together, this underlines the requirement of new model systems for the investigation of the (patho-)physiology of meibomian glands in a physiologic environment.

In the present study, we investigated the hypothesis that intercellular cohesion correlates with physiologic processes of differentiation and maturation of meibocytes. Using two independent approaches, a stable cell line of human meibocytes as well as a newly established ex vivo slice culture model of human eyelid, we identified Ecad but not desmosomal cadherins as a key player in providing intercellular cohesion, depending on the degree of differentiation. Furthermore, our ex vivo slice culture model of human eyelids may be a promising model system for detailed investigation of (patho-)physiologic processes in meibomian glands and may be suitable for preclinical studies.

\section{Materials and Methods}

\section{Cell Culture and Testing Reagents}

Cell cultures were prepared and maintained as described before for HMGECs ${ }^{4}$ and for HaCaT. ${ }^{13}$ Testing reagents were applied for the last 24 hours of each experiment. AK23 was purchased from Biozol (Eching, Germany) and used at $75 \mu \mathrm{g} / \mathrm{mL}$. Serum of patients with PV was donated by Enno Schmidt (Department of Dermatology, University of Lübeck, Lübeck, Germany) and used with the patient's informed and written consent and with institutional approval of the ethics committee (approval number 07-180). PV was diagnosed by enzyme-linked immunosorbent assay against Dsg1 (activity, $9263 \mathrm{U} / \mathrm{mL}$ ) and Dsg3 (activity, 18,383 U/mL) and by clinical and histologic phenotype. IgG fractions of patients with PV (PV-IgG) or healthy donors (control-IgG) were purified as described previously. ${ }^{14,15}$ After removal of sodium azide, monoclonal antibody against Ecad (final concentration, 8 $\mu \mathrm{g} / \mathrm{mL}$; SHE78-7, Thermo Fisher Scientific, Waltham, MA), Dsg2 (final concentration: $1 \mu \mathrm{g} / \mathrm{mL}$; clone 10G11, Progen, Heidelberg, Germany), and Dsg1 (used in a 1/50 dilution; clone p124, Progen) were used for interfering with the respective adhesion molecule.

\section{Preparation of Murine Eyelid Cryosections}

Progeny of heterozygous B6; 129X1-Dsg $3^{\text {tm1Stan } / J}$ mice (Jackson Laboratory, Bar Harbor, ME) ${ }^{16}$ were genotyped following a well-established protocol. ${ }^{17,18}$ Eyes together with eyelids of adult homozygous Dsg $3^{+/+}$(wild-type) or Dsg $3^{-/-}$ (knockout) animals were enucleated, and $7-\mu \mathrm{m}$ cryosections were prepared as described previously. ${ }^{19}$ All animal experiments were approved by the Regierung von Oberbayern (number 55.2-1-54-2532-139-2014) and complied with the Association for Research in Vision and Ophthalmology Animal Statement for the Use of Animals in Ophthalmic and Vision Research.

\section{Ex Vivo Human Eyelid Slice Model}

Tissue samples of upper eyelids were obtained from three cadavers (two females and one male) of the human body donor program of the Institute of Anatomy and Cell Biology, Ludwig-Maximilians-Universität München, Munich, Germany. For the use of samples in research, body donors gave written informed consent. This study was conducted according to the tenets of the Declaration of Helsinki. Eyelid biopsy specimens were acquired from body donors within 12 to 24 hours post mortem and were free of eye infections or known ocular diseases.

After careful removement of the overlying fat or connective tissue and epidermis, tarsal plates were embedded in $37^{\circ} \mathrm{C}$ low-melt agarose (Carl Roth, Karlsruhe, Germany) dissolved in serum-containing medium. Immediate curing of agarose was achieved by cooling on ice, and samples were cut in $300-\mu \mathrm{m}$ slices using a LeicaVT1200S vibratome (Leica Biosystems) and collected in ice-cold medium. Slices were then immediately transferred to the respective cultivation medium supplemented with the indicated testing reagent and cultivated in a constant humidified atmosphere (5\% carbon dioxide and $21 \%$ oxygen at $37^{\circ} \mathrm{C}$ ) for 24 hours. Tissue viability was ensured by MTT assay ${ }^{20}$ before slice samples were mounted in tissue freezing medium (Leica, Mannheim, Germany) and cut in $7-\mu \mathrm{m}$ section for immunostaining analyses.

\section{Immunostaining}

Immunostaining of HMGECs and cryosections were performed as described elsewhere, ${ }^{5,21}$ subjected to the respective primary antibodies anti-Ecad monoclonal antibody $(\mathrm{mAb})$ (clone 36; BD Transduction, Heidelberg, Germany), antiDsg3 polyclonal antibody (Biozol), or anti-Dsg3 mAb (Thermo Fisher Scientific; only used for murine eyelid staining) and the Cy2-labeled secondary antibodies goat antimouse or goat anti-rabbit antibodies (Dianova, Hamburg, Germany) were used. Cell nuclei were stained with DAPI (la Roche, Basel, Switzerland) and lipid accumulation with LipidTox red neutral lipid stain (Thermo Fisher Scientific).

\section{Dispase-Based Dissociation Assay}

To quantify intercellular cohesion, HMGECs were grown under the conditions indicated, and dissociation assays were performed as described for keratinocytes. ${ }^{13,14}$ Briefly, 
confluent cell monolayers were washed with Hanks' balanced salt solution and incubated with $150 \mu \mathrm{L}$ of Dispase II (2.4 U/ $\mathrm{mL}$ in Hanks' balanced salt solution; both Sigma-Aldrich, St Louis, MO) for 20 minutes at $37^{\circ} \mathrm{C}$ to detach monolayers from the well bottom. After replacement of Dispase II by $350 \mu \mathrm{L}$ of Hanks' balanced salt solution, cell monolayers were subjected to a defined mechanical stress by pipetting up and down 10 times using a 1-mL electric pipette (Eppendorf, Hamburg, Germany). Resulting fragments were stained with an MTT dye and counted using a binocular microscope (Leica) at strictly the same magnification.

\section{Protein Extraction and Western Blot Analysis}

Protein extraction and Western blot analysis were performed using standard procedures. ${ }^{22,23}$ Blots were probed with primary antibodies against desmoplakin polyclonal antibody and glyceraldehyde-3-phosphate dehydrogenase mAb (Santa Cruz Biotechnology, Dallas, TX); E-cad mAb (clone 36, BD Transduction); plakoglobin mAb, Dsg1 mAb, and Dsg2 mAb (Progen); and Dsg3 polyclonal antibody (Biozol). For protein detection, polyclonal horseradish peroxidase-conjugated goat anti-rabbit (Cell Signaling, Frankfurt, Germany) or goat anti-mouse (Dianova) IgG antibodies and an ECL reaction system (self-made solutions) were used.

\section{Lipid Quantification}

Lipid quantification was conducted with ImageJ version 1.52a (NIH, Bethesda, MD; http://imagej.nih.gov/ij). Therefore, images using RGB color space were transformed into an 8-bit format and inverted. By thresholding, artifacts were eliminated from the images. Within each independent experiment, strictly the same threshold setting was used. In these processed images, black areas represented lipid particles, whereas white areas indicated the background. With ImageJ, the total mean amount as well as the total mean size of lipid droplets were evaluated.

\section{Statistical Analysis}

Each experiment was performed at least in duplicate. Images were processed using Adobe Photoshop CS5 (Adobe, Dublin, Ireland). For densitometric measurements, the gel analysis function in ImageJ was used. Statistical analysis was performed using OriginPro 2016, 93G (Origin Lab, Northampton, MA). Significance was assumed when $P<0.05$. Data are presented as means \pm SEM. Each $n$ represents one independent experiment.

\section{Results}

Serum-Induced Differentiation Promotes the Formation of Adhesive Contacts among HMGECS

It was previously reported that in human meibomian glands in situ the presence of intercellular adhesion junctions depends on the degree of cell differentiation. ${ }^{5}$ Here, we used the stable cell line $\mathrm{HMGEC}^{10}$ to further investigate the correlation between cell maturation and the establishment of intercellular cohesion of meibocytes. We initially elaborated the optimal cultivation conditions for our in vitro studies: after cultivation in serum-free proliferation medium to approximately $90 \%$ confluence for 4 days, HMGECs were incubated for a short-term ( 1 day) to a long-term (6 days) period with serum-supplied differentiation medium (Figure 1A). Then the intracellular lipid accumulation, which is a well-established indicator of matured meibocytes, ${ }^{10}$ was analyzed by LipidTox staining (Figure 1B). Before switching to serum-containing medium, only a few lipid droplets were detectable. Serum supply for 1 day and 6 days induced synthesis of lipid vesicles, which was even more increased by adding eicosapentaenoic acid (EPA), an $\omega 3$ fatty acid important for the in vivo synthesis of human meibum. ${ }^{24,25}$ Evaluation of LipidTox staining revealed that HMGECs cultivated with serum alone displayed nearly the same amount of lipid vesicles for 1 day and 6 days, whereas the additional supply of EPA significantly increased the number of lipid vesicles. In contrast, the size of lipid vesicles was increased significantly by EPA in HMGECs after 1 day but not after 6 days compared with the respective serum-treated controls. Interestingly, the size of lipid vesicles in cells cultured in serum-free medium was comparable to serum-treated controls (Figure 1C). To investigate the effect of serum supply on cell-cell cohesion in HMGECs, dispase-based dissociation assays were performed (Figure 1D) as used before for different cultured cell types, such as keratinocytes, enterocytes, and cardiomyocytes. ${ }^{13,26,27}$ HMGECs cultivated in serum-free medium did not form confluent monolayers and disintegrated into single cells on dispase incubation, whereas serum supply for 1 day promoted intercellular cohesion, which was significantly increased after 6 days. After 6 days of treatment with serum or serum and EPA, cells detached as a monolayer from the well bottom. Interestingly, the addition of EPA for 1 day but not for 6 days resulted in a significant loss of cell-cell cohesion. Taken together, seruminduced differentiation of HMGECs is associated with intracellular lipid accumulation and establishment of firm cell-cell cohesion.

\section{Serum-Mediated Differentiation Induces the Expression of Desmosomal Components in HMGECs}

Because HMGECs form confluent, adhesive monolayers in response to the switch from serum-free to serumsupplemented medium, we hypothesized that the expression of adhesion molecules is differentiation dependent. In line with our in situ study on meibocytes, ${ }^{5}$ HMGECs displayed characteristic expression patterns of certain well-known molecules important for intercellular cohesion on serum-induced differentiation (Figure 2A). Before switching to serum, protein levels of all desmosomal components but not of Ecad at 

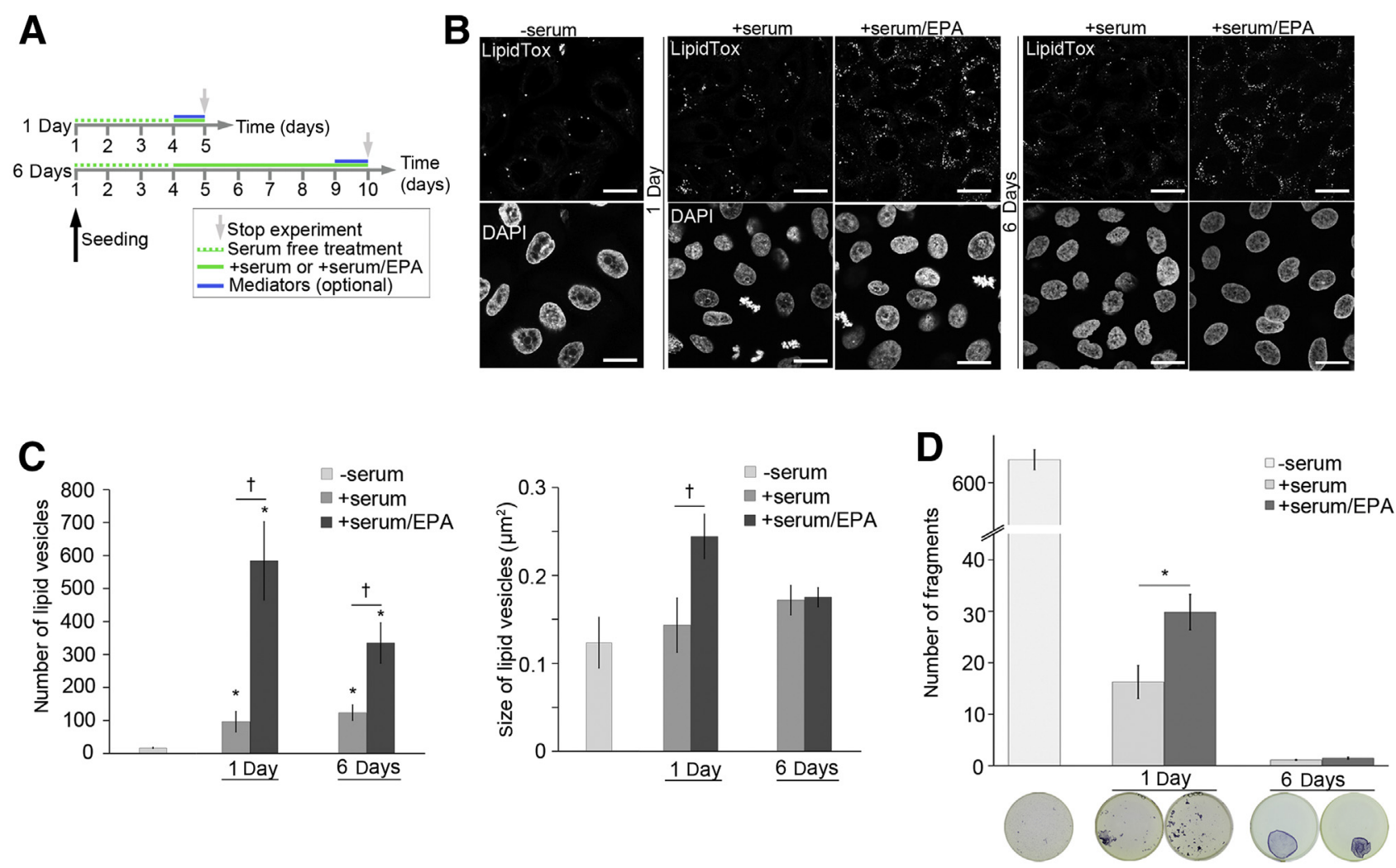

Figure 1 Serum supply induces lipid accumulation and intercellular cohesion in human meibomian gland epithelial cells (HMGECs) compared with serum-free condition. A: Overview of the cultivation conditions for HMGECs used in all experiments. B: LipidTox staining before and after switch from serum-free condition to medium supplemented with serum or serum and eicosapentaenoic acid (EPA) for 1 day or 6 days. C: Evaluation of LipidTox staining of images shown in B and indicated as the number of lipid vesicles or size of lipid vesicles. D: Dispase-based dissociation assay for quantification of intercellular cohesion. Representative images of cell monolayers after mechanical shear stress. $n=5$ (B and $\mathbf{C}) ; n=7$ (D). ${ }^{*} P<0.05$ versus without serum treatment; ${ }^{\dagger} P<0.05$. Scale bars $=10 \mu \mathrm{m}$.

days 1 and 6 as well as of Dsg1 at day 6 were very low. Parallel to enhanced intercellular cohesion, serum supply for 1 day or 6 days increased the protein levels of all analyzed adhesion molecules, whereas addition of EPA reduced significantly the protein amounts of desmoplakin, Dsg3, and plakoglobin after 1 day but not after 6 days. This observation was also supported by immunostaining experiments against Dsg3 and Ecad (Figure 2B). HMGECs cultivated in serumfree medium for 1 day or 6 days displayed, albeit weakly after 1 day, membrane Ecad staining, whereas Dsg3 was hardly detectable. Again, the supply of serum or serum and EPA enhanced localization of both Ecad and Dsg3 along cell membranes after 1 day. Interestingly, 6 days of treatment with serum or serum and EPA resulted in increased membrane Dsg3 but not Ecad staining. This finding indicates that in HMGECs both expression and membrane localization of adhesion molecules, such as Ecad and Dsg3, are regulated in a differentiation-dependent manner.

\section{Inhibition of Ecad but Not of Desmosomal Cadherins Drastically Impairs Intercellular Cohesion and Affects Intracellular Lipid Accumulation of HMGECs}

To further analyze the correlation between differentiation and the formation of strong adhesive contacts in HMGECs, dispase-based dissociation assays were performed after blocking the interactions of certain adhesion molecules by inhibitory antibodies (Figure 3A). Because Dsg3-deficient (Dsg3 knockout) mice not only display a PV phenotype with lesions in the $\operatorname{skin}^{16,28}$ but also develop conjunctional blistering, ${ }^{19}$ the effect of AK23, a monoclonal pemphigus mouse model antibody against Dsg3, ${ }^{29}$ and PV-IgG was first studied with autoantibodies against Dsg1 and Dsg3 from a patient with PV. Surprisingly, and in contrast to previous observations in human and mouse keratinocytes, ${ }^{13,14,17}$ neither AK23 nor PV-IgG significantly affected intercellular cohesion in HMGECs. The efficiency of AK23 and PV-IgG used in the present study was verified by dissociation assays performed in human keratinocytes (Supplemental Figure S1). Similar to AK23 and PV-IgG, purchased antibodies that targeted Dsg1 and Dsg2, which were previously proven to be inhibitory by atomic force microscopy and dissociation assays ${ }^{26,30}$ also had no effect on dispase-based dissociation assays.

However, in line with previous observations in keratinocytes, ${ }^{23}$ antibody-mediated inhibition of Ecad binding drastically impaired intercellular cohesion after 1 day. Indeed, HMGECs disintegrated, similar to cells cultivated in serum-free medium, into single cells. Interestingly, after 6 days, HMGECs appeared to be resistant against the 
A
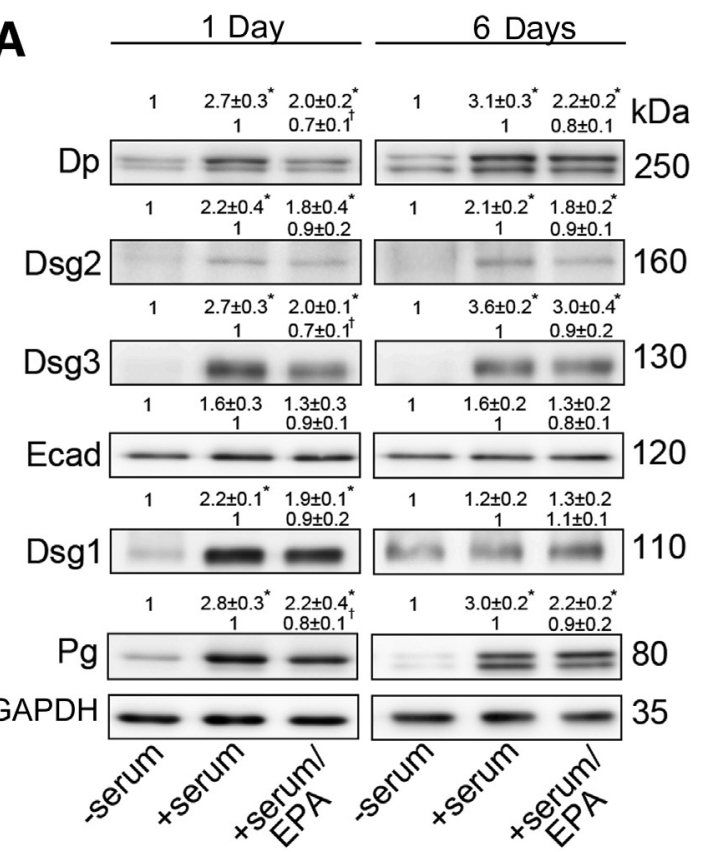

B
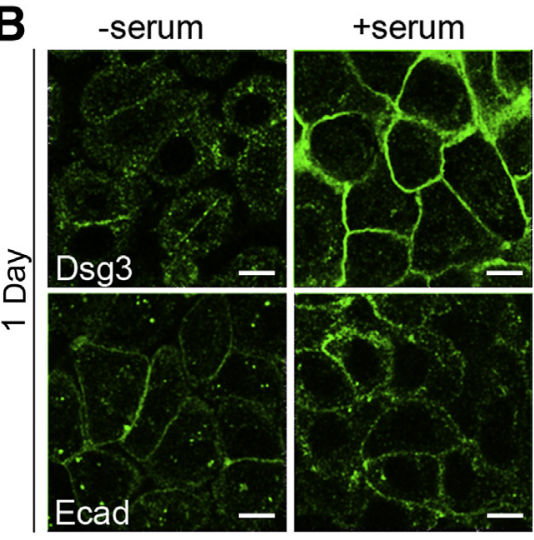

+serum/EPA
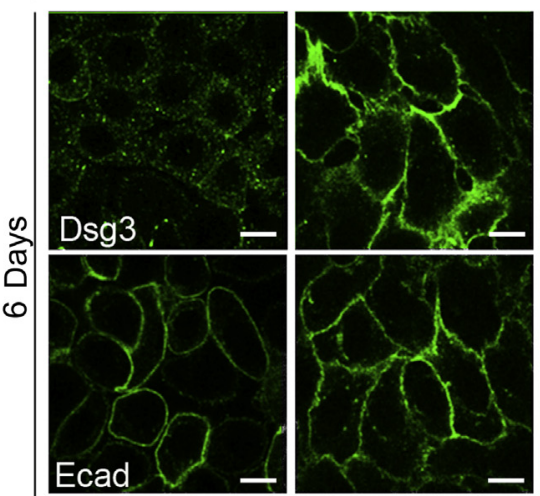

Figure 2 Serum supply promotes expression of certain adhesion molecules in human meibomian gland epithelial cells. A: Representative Western blot of a set of adhesion molecules at days 1 and 6 before and after switch from serum-free condition to medium supplemented with serum or serum and eicosapentaenoic acid (EPA). Protein levels are quantified and indicated above each Western blot line as fold of without serum treatment (upper values) or as fold of with serum treatment (lower values). B: Immunostaining against desmoglein (Dsg) 3 or E-cadherin (Ecad) was performed in parallel to Western blot analyses. $n=4$. ${ }^{*} P<0.05$ versus without serum treatment; ${ }^{\dagger} P<0.05$ versus with serum treatment. Scale bar $=10 \mu \mathrm{m}$. Dp, desmoplakin; GAPDH, glyceraldehyde-3-phosphate dehydrogenase; $\mathrm{Pg}$, plakoglobin. antibody-mediated impairment of Ecad binding. Only the combination of antibodies that targeted Ecad and Dsg3 by AK23 increased the number of fragments significantly when HMGECs were cultivated with serum and EPA but not with serum alone. These findings were further corroborated by immunostaining analyses performed in parallel to dispasebased assays (Figure 3B). The confluency of cell monolayers and the linear staining of Ecad or Dsg3 was initially confirmed at cell junctions in controls. In 1-day cultures, antibody-mediated targeting of Ecad resulted in formation of intercellular gaps (arrows), which was not observed after AK23 treatment. In 6-day cultures, no gap formation as well as no effect on Dsg3 or Ecad staining were induced by AK23 or anti-Ecad antibody.

Furthermore, we were interested whether cell cohesion regulates lipid production in meibocytes. Quantification of LipidTox staining (Figure 3C) revealed that the antibodymediated targeting of Ecad significantly reduced the number of lipid vesicles in HMGECs cultivated with serum or serum and EPA for 1 day compared with controls. Nevertheless, under this condition, an increased amount of lipid vesicle was still inducible by the supply of EPA. In contrast, accumulation of intracellular lipid vesicles was not significantly altered by AK23 treatment compared with the respective controls. Interestingly, in 6-day cultures, antibody-mediated targeting of Ecad but not of Dsg3 reduced the number of vesicles only in HMGECs cultivated with serum and EPA. Together, although HMGECs express both the main adhesion molecules of AJs and desmosomes in differentiation-dependent manner, our findings indicate a central role of Ecad but not of Dsg1 and Dsg3 for intercellular adhesion and lipid synthesis in HMGECs.

\section{Impairment of Ecad but Not of Dsg3 Binding Affects Lipid Accumulation in a Human ex Vivo Eyelid Model}

To further substantiate the impact of intercellular cohesion on maturation and differentiation processes and lipid accumulation in meibocytes, a new ex vivo slice culture model of human eyelids was established. Here, human eyelid slices were incubated with either anti-Ecad antibody or AK23 in medium that contained serum or serum and EPA for 1 day. Tissue viability of eyelid biopsy specimens was tested by MTT assay (Supplemental Figure S2A) before cryosections of the cultivated human eyelid slices were stained against Ecad and lipid vesicle were labeled with LipidTox. Lipid staining was quantified as described in Supplemental Figure S2B.

No changes of staining intensity or intracellular distribution of Ecad were detectable under the indicated culture conditions (Figure 4A). To rule out unspecific signals, secondary antibody controls were stained in parallel (Supplemental Figure S2C). In contrast to Ecad staining, LipidTox staining intensity seemed to be modulated, depending on the respective cultivation condition. This observation was further corroborated by quantitative 
A

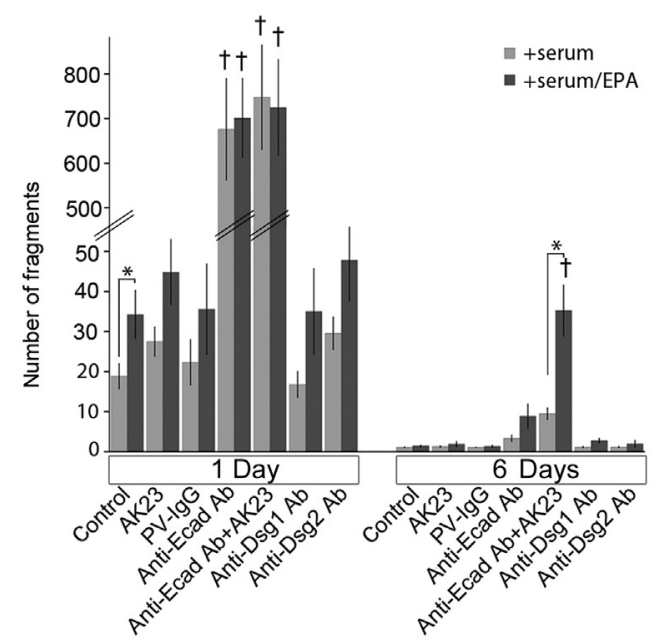

B
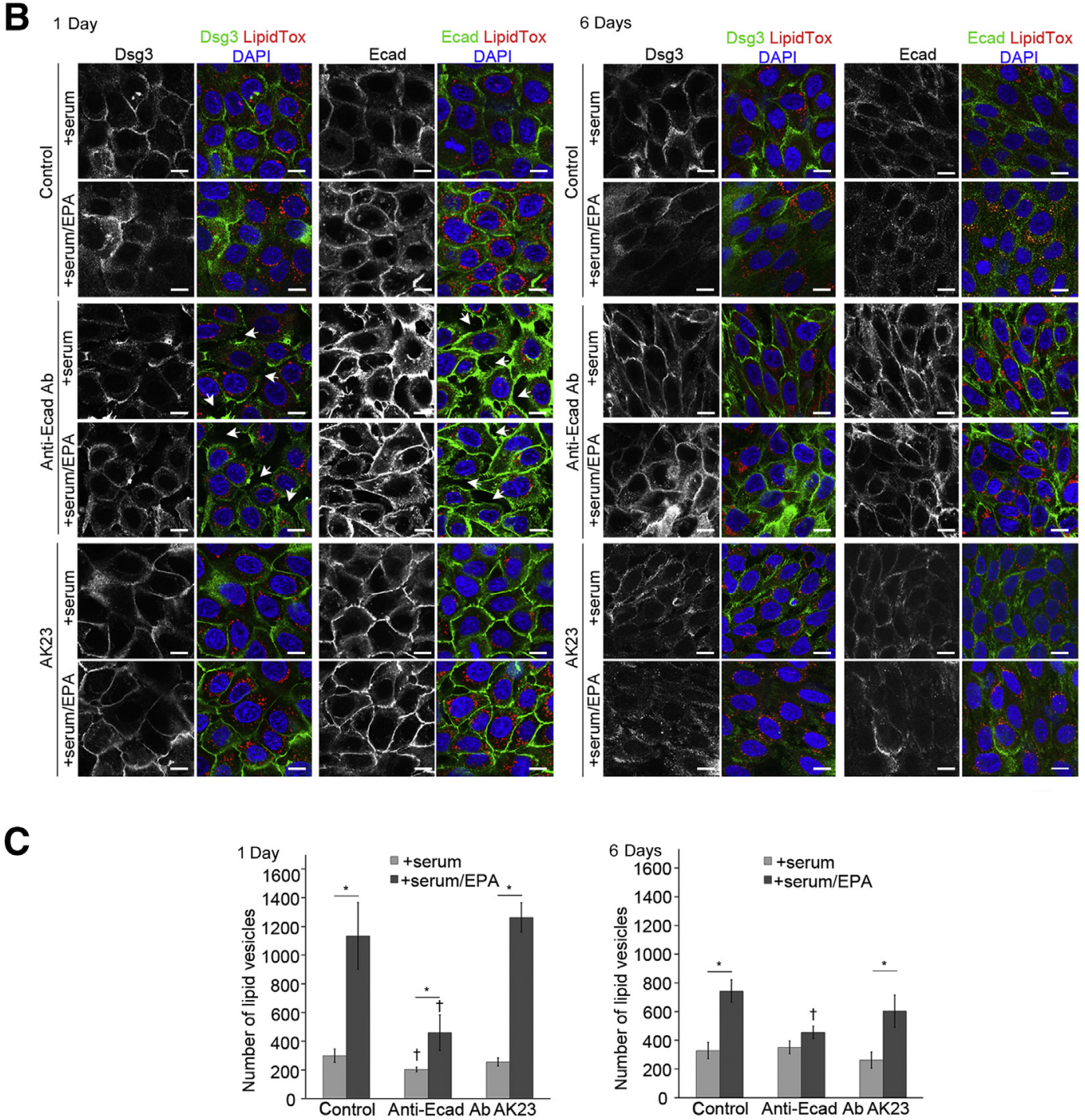

Figure 3 Inhibition of E-cadherin (Ecad) but not of desmosomal proteins compromises the formation of adhesive monolayers and lipid accumulation in human meibomian gland epithelial cells. A: Dispase-based dissociation assay after switch to medium that contains serum or serum and eicosapentaenoic acid (EPA) and parallel incubation with inhibitory antibodies (Abs) that target desmoglein (Dsg) 3, Ecad, Dsg1, and Dsg2. B: Immunostaining against Ecad and Dsg3 and LipidTox labeling after treatment with IgG fractions of healthy donors (control-IgG), anti-Ecad Ab, or AK23, a pathogenic Ab directed against Dsg3. Arrows indicate gaps within cell monolayers. C: Quantification of LipidTox staining in images shown in B. $n=8(\mathbf{A}) ; n=5(\mathbf{B})$. ${ }^{*} P<0.05 ;{ }^{\dagger} P<0.05$ versus respective control. Scale bar $=10 \mu \mathrm{m}$. 
A

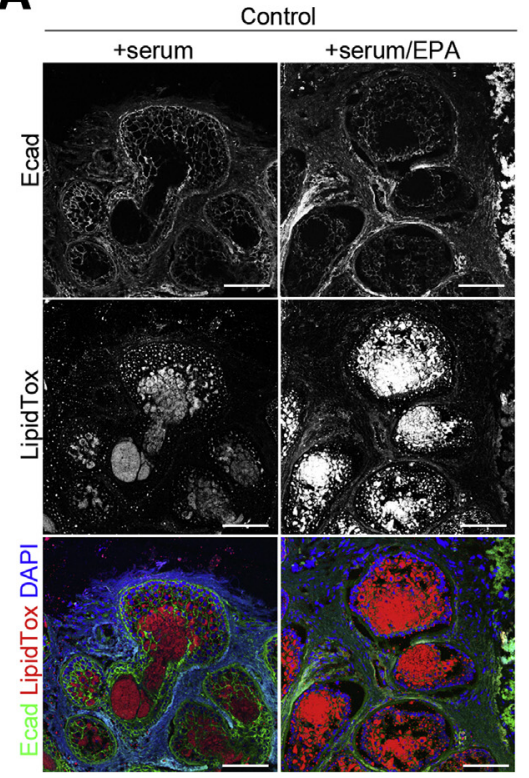

B

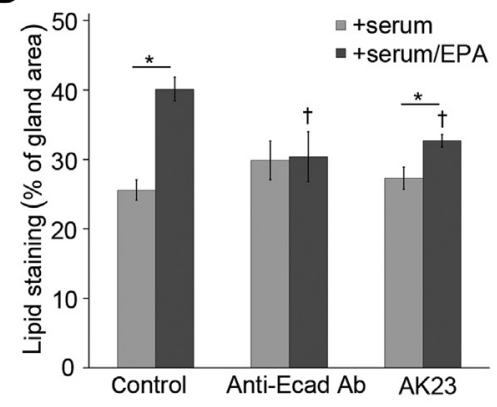

D

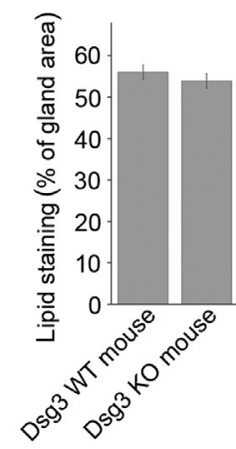

Anti-Ecad Ab
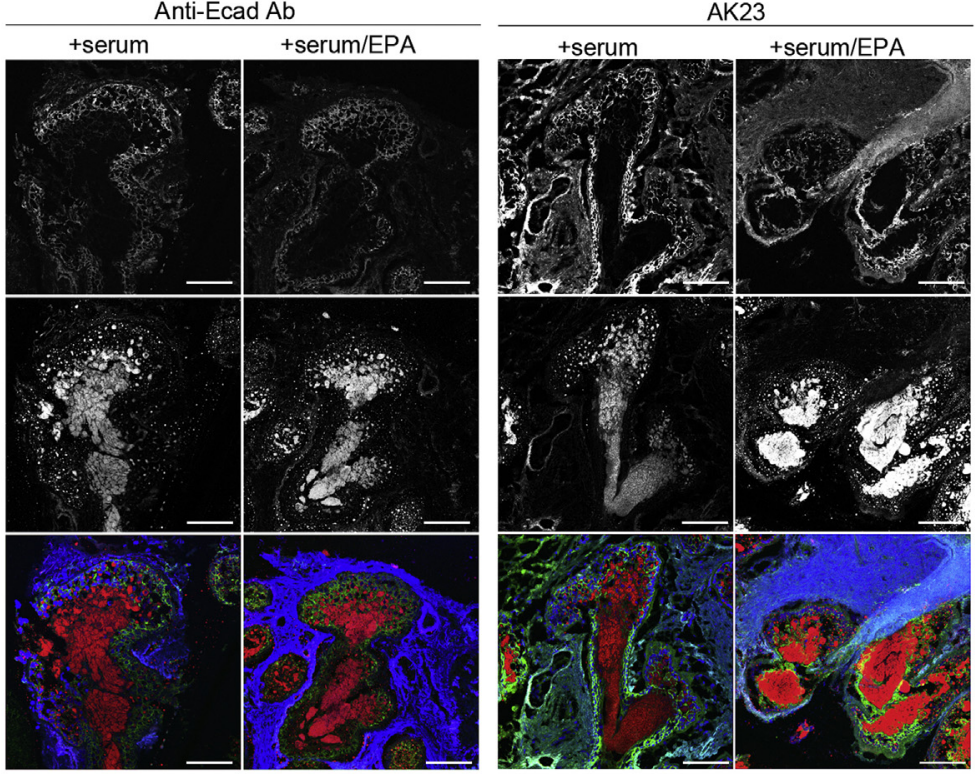

C
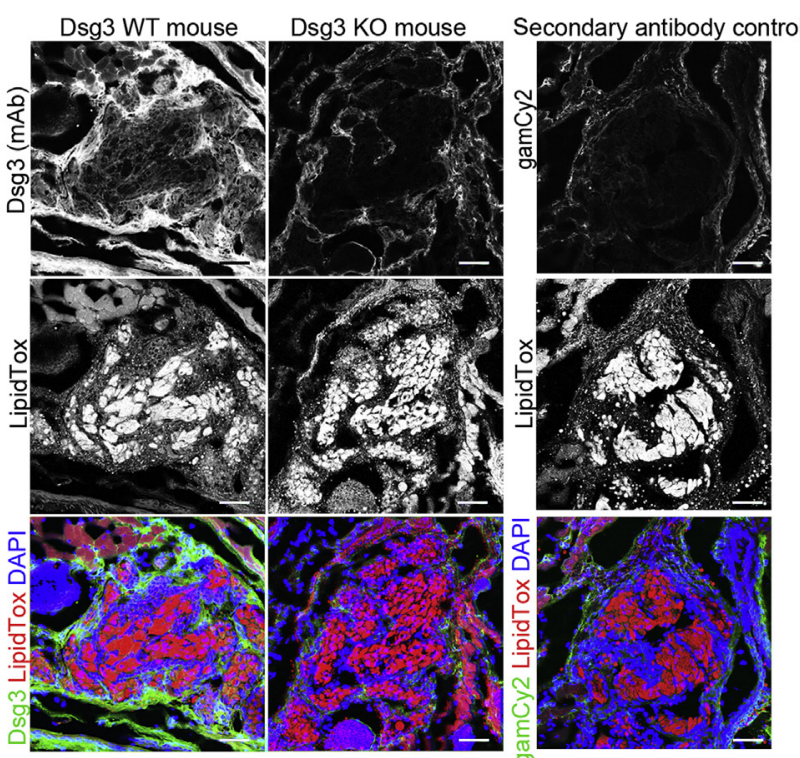

Figure 4 Impairment of E-cadherin (Ecad) but not of desmoglein (Dsg) 3 binding affects lipid accumulation in human ex vivo eyelid models. A: Ecad staining in human eyelid slices treated with IgG fractions of healthy donors (control-IgG), anti-Ecad antibody (Ab), or AK23 in medium supplemented with serum or serum and eicosapentaenoic acid (EPA) for 1 day. B: Quantification of LipidTox staining in images shown in A. C: Ecad staining in eyelid sections of Dsg3 wild-type (WT) and Dsg3 knockout (KO) mice. Ab specificity is proven by secondary only controls. D: Quantification of LipidTox staining in images shown in $\mathbf{C}$. $n=6(\mathbf{A}$ and $\mathbf{B}) ; n=$ 3 (C and $\mathbf{D}) .{ }^{*} P<0.05 ;{ }^{\dagger} P<0.05$ versus respective control. Scale bars: $100 \mu \mathrm{m}(\mathbf{A}) ; 50 \mu \mathrm{m}(\mathbf{C})$.

analyses of lipid staining (Figure 4B). Similar to HMGECs, meibomian gland cells of the ex vivo human eyelid slice culture model accumulated significantly more lipid vesicles when cultured in serum and EPA compared with serum alone. Moreover, antibody-mediated Ecad targeting abolished the serum- and EPA-induced increase of lipid staining in the eyelid slice culture model. Interestingly, this incapacity of meibomian gland cells treated with inhibitory anti-Ecad antibody to accumulate lipid vesicles in response to serum and EPA supply was similar to the effects described above for HMGECs after 6 days. Furthermore, AK23-treated meibomian gland cells of our ex vivo human eyelid slice culture model displayed a significantly increased amount of lipid staining after serum and EPA treatment compared with the respective serum condition, which is also in line with cell culture data.

Vice versa, to study whether loss of Dsg3 modulates lipid accumulation in meibomian glands, lipid accumulation in 
eyelid sections of Dsg3 knockout mice was analyzed. Compared with their wild-type littermates, Dsg3 knockout mice did not display any differences in gland morphologic features or in lipid staining (Figure 4C). Quantification of lipid staining further confirmed that the loss of Dsg3 did not impair accumulation of lipid vesicles within murine meibomian gland cells (Figure 4D).

\section{Discussion}

We studied for the first time the regulation of cell cohesion and its interplay with lipid production in meibomian glands. This study provides new evidence that the formation of cellcell contacts as well as the synthesis of lipids are dependent on the degree of differentiation and shows that Ecad rather than adhesion molecules of desmosomes is essential for these physiologic processes in meibocytes. In addition, we established a novel ex vivo model of human eyelids, which confirmed the in vitro data of the present study but also may be a promising approach for further detailed analyses of (patho-)physiologic processes in meibomian glands as well as for identification and testing of MGD-related pharmaceuticals in preclinical studies.

\section{Ecad Is Important for Cell Cohesion and Lipid Production in Meibocytes}

Serum-induced stimulation of differentiation-associated processes is well established for many types of epithelial cells $^{31-33}$ and in particular for HMGECs. ${ }^{4,10}$ Using this stable cell line of HMGECs, we demonstrated a serummediated induction of intercellular cohesion that increases over time as well as a specific differentiation-dependent expression pattern of adhesion molecules important for strong intercellular contacts. We hypothesized that seruminduced intercellular cohesion of meibocytes was correlated with the increased levels of desmosomal cadherins observed after serum treatment, which would have been in line with our in situ data of human eyelids demonstrating an increased number of desmosomes in matured compared with basal meibocytes. ${ }^{5}$ However, based on data from the current study, Ecad was identified as a key player in meibocyte differentiation. Thus, antibody-mediated interference with Ecad but not with Dsg3 resulted in i) morphologic changes, ii) severely impaired intercellular cohesion, and iii) significantly reduced intracellular lipid accumulation of HMGECs after 1 day but not after 6 days of differentiation. In addition, among the investigated adhesion proteins, Ecad was the only molecule in meibocytes expressed not only under differentiation but also under proliferation conditions. Because proliferating HMGECs disintegrated into single cells on mechanical shear stress, Ecad may have additional functions different from providing intercellular cohesion. An active crosstalk between the adhesion molecules of AJs and desmosomes is well established, ${ }^{34-36}$ and in keratinocytes, a signaling complex between Dsg3 and $\mathrm{Ecad}^{23}$ as well as between Dsg2 and Ecad ${ }^{37}$ was identified recently to modulate desmosomal assembly and adhesion. On the basis of this finding, it is possible that in HMGECs Ecad serves as a prerequisite for the recruitment of desmosomal cadherins to the cell membrane, thereby facilitating the formation of desmosomes. In addition, our finding that HMGECs cultivated for 6 days but not for 1 day were resistant against mechanical stress after treatment with pathogenic anti-Ecad antibody further supports an important role of Ecad for cell cohesion during early stages of differentiation.

\section{The Human ex Vivo Human Eyelid Model Is Suitable to Study (Patho-)Physiologic Processes in Meibomian Glands}

Because the architecture of meibomian gland acini as well as the underlying processes of cell differentiation are highly complex, it is questionable whether the conventional twodimensional cell culture of HMGECs is fully sufficient to study the physiology of meibocytes and the pathomechanisms of MGD. Recent attempts of establishing a suitable in vitro three-dimensional meibomian culture model also faced limitations. ${ }^{11}$ Together with the lack of appropriate animal models, ${ }^{11,12}$ we are aware of the urgent requirement of an appropriate model system to study the (patho-)physiology of meibomian glands. Therefore, recently established ex vivo slice culture models were adapted to study cell cohesion in human skin and murine heart ${ }^{27,38}$ and established a novel ex vivo slice culture model of human eyelids. Importantly, meibocytes in our eyelid slice model were viable after 1-day cultivation and showed remarkable similarities to HMGECs after 6-day differentiation. The in vitro as well as the ex vivo approach demonstrated that lipid production was not inducible by the supply of EPA in meibocytes on antibody-mediated interference of Ecad binding. Thus, our ex vivo slice culture model of human eyelids not only confirmed in vitro data of the present study but may also be suitable for detailed analyses of (patho-) physiologic processes of meibocytes in a more physiologic environment in the future.

\section{The Role of MGD for Ocular Involvement in Pemphigus}

A deepened understanding of the impact of intercellular cohesion among meibocytes during differentiation and the associated processes is of relevance because an increasing number of case reports also described eye involvement in patients with PV. ${ }^{39-41}$ In a broad case study from 2015, Tan et $\mathrm{al}^{7}$ further identified a high prevalence of DED in patients with PV. Here as well as in the recent in situ study, ${ }^{5}$ we demonstrated that meibocytes display a composition of adhesion molecules similar to the epidermis, including Dsg 1 and Dsg3 as the primary targets of PV autoantibodies. ${ }^{42}$ However, using antibodies against Dsg1 and Dsg3, we 
demonstrated that in meibocytes neither cell-cell cohesion nor lipid production is regulated by desmosomal adhesion. Given the correlation between DED and pemphigus, our data suggest that ocular involvement in pemphigus is not caused primarily via MGD but rather via direct effects of autoantibodies on human conjunctiva as previously reported. ${ }^{19}$

Nevertheless, besides direct impairment of desmosomal adhesion, it is well established that binding of pathogenic PV antibody modulates numerous intercellular signaling pathways (eg, p38 mitogen-activated protein kinase and protein kinase C). ${ }^{42,43}$ Therefore, it cannot currently be ruled out that autoantibodies in pemphigus interfere with signaling pathways required for physiologic processes of meibocytes besides cell adhesion and lipid production. Because ocular pemphigus still remains underdiagnosed $d^{7,39}$ and no eye biopsy specimens of patients with PV are available, the novel ex vivo slice culture model of human eyelids may be a promising model for further studies on the signaling pathways involved in PV pathogenesis. Moreover, the model may provide new insights into the role of intercellular cohesion and its regulation, which ultimately may also allow the establishment and testing of new therapeutic approaches for treatment of MGD-associated diseases.

\section{Acknowledgments}

We thank Martina Hitzenbichler for her excellent technical assistance, Jana Daimer, Jessica Plewa, Michael Becker, and Axel Unverzagt for their skillful help in preparation of human body donors' eyelids, and Enno Schmidt for donating serum of patients with PV.

V.R. designed the study, conceived and performed experiments, and wrote the manuscript; F.M. performed experiments and analyzed data; F.G. and F.P. interpreted data and wrote the manuscript; J.W. designed and supervised the study; all authors wrote the manuscript and had final approval of the submitted and published versions.

\section{Supplemental Data}

Supplemental material for this article can be found at http://doi.org/10.1016/j.ajpath.2019.04.015.

\section{References}

1. Nelson JD, Shimazaki J, Benitez-del-Castillo JM, Craig JP, McCulley JP, Den S, Foulks GN: The international workshop on meibomian gland dysfunction: report of the definition and classification subcommittee. Invest Ophthalmol Vis Sci 2011, 52:1930-1937

2. Nichols KK: The international workshop on meibomian gland dysfunction: introduction. Invest Ophthalmol Vis Sci 2011, 52: 1917-1921

3. Knop E, Knop N, Millar T, Obata H, Sullivan DA: The international workshop on meibomian gland dysfunction: report of the subcommittee on anatomy, physiology, and pathophysiology of the meibomian gland. Invest Ophthalmol Vis Sci 2011, 52:1938-1978
4. Hampel U, Schroder A, Mitchell T, Brown S, Snikeris P, Garreis F, Kunnen C, Willcox M, Paulsen F: Serum-induced keratinization processes in an immortalized human meibomian gland epithelial cell line. PLoS One 2015, 10:e128096

5. Rötzer V, Egu D, Waschke J: Meibomian gland cells display a differentiation-dependent composition of desmosomes. Histochem Cell Biol 2016, 146:685-694

6. Stanley JR, Amagai M: Pemphigus, bullous impetigo, and the staphylococcal scalded-skin syndrome. N Engl J Med 2006, 355: $1800-1810$

7. Tan JC, Tat LT, Francis KB, Mendoza CG, Murrell DF, Coroneo MT: Prospective study of ocular manifestations of pemphigus and bullous pemphigoid identifies a high prevalence of dry eye syndrome. Cornea 2015, 34:443-448

8. Spindler V, Eming R, Schmidt E, Amagai M, Grando S, Jonkman MF, Kowalczyk AP, Muller EJ, Payne AS, Pincelli C, Sinha AA, Sprecher E, Zillikens D, Hertl M, Waschke J: Mechanisms causing loss of keratinocyte cohesion in pemphigus. J Invest Dermatol 2018, 138:32-37

9. Spindler V, Waschke J: Pemphigus: a disease of desmosome dysfunction caused by multiple mechanisms. Front Immunol 2018, 9:136

10. Sullivan DA, Liu Y, Kam WR, Ding J, Green KM, Shaffer SA, Hatton MP, Liu S: Serum-induced differentiation of human meibomian gland epithelial cells. Invest Ophthalmol Vis Sci 2014, 55: 3866-3877

11. Asano N, Hampel U, Garreis F, Schroder A, Schicht M, Hammer CM, Paulsen F: Differentiation patterns of immortalized human meibomian gland epithelial cells in three-dimensional culture. Invest Ophthalmol Vis Sci 2018, 59:1343-1353

12. Tong L, Gupta PK: Need for animal models of meibomian gland dysfunction. Ophthalmol Ther 2016, 5:129-134

13. Rötzer V, Breit A, Waschke J, Spindler V: Adducin is required for desmosomal cohesion in keratinocytes. J Biol Chem 2014, 289: 14925-14940

14. Spindler V, Rötzer V, Dehner C, Kempf B, Gliem M, Radeva M, Hartlieb E, Harms GS, Schmidt E, Waschke J: Peptide-mediated desmoglein 3 crosslinking prevents pemphigus vulgaris autoantibodyinduced skin blistering. J Clin Invest 2013, 123:800-811

15. Heupel WM, Zillikens D, Drenckhahn D, Waschke J: Pemphigus vulgaris $\operatorname{Ig} \mathrm{G}$ directly inhibit desmoglein 3-mediated transinteraction. J Immunol 2008, 181:1825-1834

16. Koch PJ, Mahoney MG, Ishikawa H, Pulkkinen L, Uitto J, Shultz L, Murphy GF, Whitaker-Menezes D, Stanley JR: Targeted disruption of the pemphigus vulgaris antigen (desmoglein 3) gene in mice causes loss of keratinocyte cell adhesion with a phenotype similar to pemphigus vulgaris. J Cell Biol 1997, 137:1091-1102

17. Hartlieb E, Rötzer V, Radeva M, Spindler V, Waschke J: Desmoglein 2 compensates for desmoglein 3 but does not control cell adhesion via regulation of p38 mitogen-activated protein kinase in keratinocytes. J Biol Chem 2014, 289:17043-17053

18. Mahoney MG, Wang Z, Rothenberger K, Koch PJ, Amagai M, Stanley JR: Explanations for the clinical and microscopic localization of lesions in pemphigus foliaceus and vulgaris. J Clin Invest 1999, $103: 461-468$

19. Vielmuth F, Rötzer V, Hartlieb E, Hirneiss C, Waschke J, Spindler V: Pemphigus autoantibodies induce blistering in human conjunctiva. Invest Ophthalmol Vis Sci 2016, 57:4442-4449

20. Mosmann T: Rapid colorimetric assay for cellular growth and survival: application to proliferation and cytotoxicity assays. J Immunol Methods 1983, 65:55-63

21. Spindler V, Drenckhahn D, Zillikens D, Waschke J: Pemphigus IgG causes skin splitting in the presence of both desmoglein 1 and desmoglein 3. Am J Pathol 2007, 171:906-916

22. Gliem M, Heupel WM, Spindler V, Harms GS, Waschke J: Actin reorganization contributes to loss of cell adhesion in pemphigus vulgaris. Am J Physiol Cell Physiol 2010, 299:C606-C613 
23. Rötzer V, Hartlieb E, Vielmuth F, Gliem M, Spindler V, Waschke J: E-cadherin and Src associate with extradesmosomal Dsg3 and modulate desmosome assembly and adhesion. Cell Mol Life Sci 2015, 72:4885-4897

24. Butovich IA, Uchiyama E, Di Pascuale MA, McCulley JP: Liquid chromatography-mass spectrometric analysis of lipids present in human meibomian gland secretions. Lipids 2007, 42:765-776

25. Hampel U, Kruger M, Kunnen C, Garreis F, Willcox M, Paulsen F: In vitro effects of docosahexaenoic and eicosapentaenoic acid on human meibomian gland epithelial cells. Exp Eye Res 2015, 140: $139-148$

26. Ungewiss H, Rötzer V, Meir M, Fey C, Diefenbacher M, Schlegel N, Waschke J: Dsg2 via Src-mediated transactivation shapes EGFR signaling towards cell adhesion. Cell Mol Life Sci 2018, 75: $4251-4268$

27. Schinner C, Vielmuth F, Rötzer V, Hiermaier M, Radeva MY, Co TK, Hartlieb E, Schmidt A, Imhof A, Messoudi A, Horn A, Schlipp A, Spindler V, Waschke J: Adrenergic signaling strengthens cardiac myocyte cohesion. Circ Res 2017, 120:1305-1317

28. Rötzer V, Hartlieb E, Winkler J, Walter E, Schlipp A, Sardy M, Spindler V, Waschke J: Desmoglein 3-dependent signaling regulates keratinocyte migration and wound healing. J Invest Dermatol 2016, 136:301-310

29. Tsunoda K, Ota T, Aoki M, Yamada T, Nagai T, Nakagawa T, Koyasu S, Nishikawa T, Amagai M: Induction of pemphigus phenotype by a mouse monoclonal antibody against the amino-terminal adhesive interface of desmoglein 3. J Immunol 2003, 170:2170-2178

30. Waschke J, Bruggeman P, Baumgartner W, Zillikens D, Drenckhahn D: Pemphigus foliaceus $\operatorname{IgG}$ causes dissociation of desmoglein 1-containing junctions without blocking desmoglein 1 transinteraction. J Clin Invest 2005, 115:3157-3165

31. Bertolero F, Kaighn ME, Camalier RF, Saffiotti U: Effects of serum and serum-derived factors on growth and differentiation of mouse keratinocytes. In Vitro Cell Dev Biol 1986, 22:423-428

32. Lechner JF, Haugen A, McClendon IA, Shamsuddin AM: Induction of squamous differentiation of normal human bronchial epithelial cells by small amounts of serum. Differentiation 1984, 25:229-237
33. Hesterberg TW, Maness SC, Iglehart JD, Sanchez JH, Boreiko CJ: Subpopulations of human bronchial epithelial cells in culture respond heterogeneously to 12-O-tetradecanoylphorbol-13-acetate (TPA) and other modulators of differentiation. Carcinogenesis 1987, 8: $1511-1515$

34. Gumbiner B, Stevenson B, Grimaldi A: The role of the cell adhesion molecule uvomorulin in the formation and maintenance of the epithelial junctional complex. J Cell Biol 1988, 107: $1575-1587$

35. Lewis JE, Wahl JK 3rd, Sass KM, Jensen PJ, Johnson KR, Wheelock MJ: Cross-talk between adherens junctions and desmosomes depends on plakoglobin. J Cell Biol 1997, 136:919-934

36. Lewis JE, Jensen PJ, Wheelock MJ: Cadherin function is required for human keratinocytes to assemble desmosomes and stratify in response to calcium. J Invest Dermatol 1994, 102:870-877

37. Shafraz O, Rubsam M, Stahley SN, Caldara AL, Kowalczyk AP, Niessen CM, Sivasankar S: E-cadherin binds to desmoglein to facilitate desmosome assembly. Elife 2018, 7:e37629

38. Egu DT, Walter E, Spindler V, Waschke J: Inhibition of p38MAPK signalling prevents epidermal blistering and alterations of desmosome structure induced by pemphigus autoantibodies in human epidermis. Br J Dermatol 2017, 177:1612-1618

39. Daoud YJ, Cervantes R, Foster CS, Ahmed AR: Ocular pemphigus. J Am Acad Dermatol 2005, 53:585-590

40. Akhyani M, Keshtkar-Jafari A, Chams-Davatchi C, Lajevardi V, Beigi S, Aghazadeh N, Rayati Damavandi M, Arami S: Ocular involvement in pemphigus vulgaris. J Dermatol 2014, 41: $618-621$

41. Broussard KC, Leung TG, Moradi A, Thorne JE, Fine JD: Autoimmune bullous diseases with skin and eye involvement: cicatricial pemphigoid, pemphigus vulgaris, and pemphigus paraneoplastica. Clin Dermatol 2016, 34:205-213

42. Waschke J: The desmosome and pemphigus. Histochem Cell Biol 2008, 130:21-54

43. Kitajima Y: 150 anniversary series: desmosomes and autoimmune disease, perspective of dynamic desmosome remodeling and its impairments in pemphigus. Cell Commun Adhes 2014, 21:269-280 